\title{
Is what's good for the gander good for the goose?
}

\author{
Nanette K. Wenger, MD
}

\begin{abstract}
Editorial Note: The gender initiative, a provocative series on gender differences in the surgical treatment of cardiac, vascular, and thoracic disease, continues this month with editorials on coronary revascularization in women.
\end{abstract}

A foremost expert in gender-specific risks and outcomes, Nanette $\mathrm{K}$. Wenger, MD, summarizes the best available data, progress to date, and remaining questions for women undergoing coronary revascularization. Another group summarizes the statistical problems that may confound studies of gender differences. Finally, cardiac surgeons contemplate possible differences in the technical aspects of coronary artery bypass grafting. The series continues in December with editorials on coronary artery surgery and recovery.

Nancy A. Nussmeier, MD Texas Heart Institute

See related editorials on pages 932, 936, 950, and 959.

\footnotetext{
Professor of Medicine (Cardiology), Emory University School of Medicine; Chief of Cardiology, Grady Memorial Hospital; Consultant, Emory Heart \& Vascular Center, Atlanta, Ga.

Received for publication May 7, 2003; accepted for publication June 3, 2003.

Address for reprints: Nanette K. Wenger, MD, Emory University School of Medicine; 69 Jesse Hill Jr Dr, SE, Atlanta, GA 30303 (E-mail: nwenger@emory.edu).

J Thorac Cardiovasc Surg 2003;126:929-31

Copyright () 2003 by The American Association for Thoracic Surgery

$0022-5223 / 2003 \$ 30.00+0$

doi:10.1016/S0022-5223(03)00883-3
}

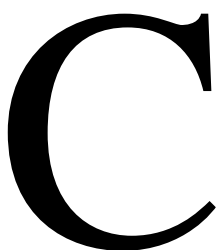

oronary heart disease is the leading cause of death for women in the United States, accounting for almost 250,000 deaths annually. Since 1984 more US women than men have died each year of cardiovascular disease. Emerging data have reinforced sex-related differences in responses to therapies, including adverse responses. This editorial highlights the excess mortality of coronary artery bypass graft $(\mathrm{CABG})$ surgery in women, addressing the need to derive more detailed sex-specific information that has the potential to improve clinical outcomes. Owing to the paucity of randomized trial data for women, the material presented derives primarily from databases, registries, and case series.

\section{What We Know}

Rates of performance of CABG surgery in the Coronary Artery Surgery Study (CASS) Registry, when adjusted for clinical and angiographic characteristics, were comparable for women and men. There were few sex differences in long-term survival, differing only by the variance in operative mortality, $5.3 \%$ for women versus $2.5 \%$ for men. ${ }^{1}$ Details of the operative mortality characteristics are not presented. Can they be derived from this data set? If not, would systematic medical record review of fatalities be feasible? As in other settings, conventional coronary risk reduction was likely suboptimal, ${ }^{2}$ a problem also common to men after CABG surgery until very recent years.

The Society of Thoracic Surgeons National Cardiac Surgery Database, which retrospectively examined 334,913 patients undergoing CABG surgery since 1994, provided important sex-based data. ${ }^{3}$ Women, who constituted $28 \%$ of the reported population, had an operative mortality of $4.5 \%$ compared with $2.6 \%$ for men $(P<$ $.0001)$. Although the mortality rate remained significantly higher for women for each of the multiple risk factors examined univariately, multivariate analysis showed that women continued to have higher mortality than equally matched men in the low and medium risk spectrum. Only among very high risk patients was there a sex-neutral mortality risk; patients at highest risk were elderly individuals, those undergoing reoperation, and those having nonelective procedures. Greater mortality risk for women persisted even after consideration of body surface area (a surrogate for coronary artery size) and the use of internal thoracic artery grafting. Despite comments that both immediate postoperative mortality and morbidity are higher in women and that long-term survival is lower, specifics of the operative mortality are not offered. It is this examination of situation- and cause-specific mortality that might more precisely identify remediable problems.

By contrast, a report from the Bypass Angioplasty Revascularization Investigation (BARI) ${ }^{4}$ showed comparable unadjusted 5-year mortality rates for women and men; with correction for the higher risk profiles of women, female sex was described as an independent predictor of improved 5-year survival. This does not, however, warrant interpretation that women do better, only that sex per se is not the feature responsible for increased mortality. BARI represents a small subset of CABG 
patients, specifically those eligible for either percutaneous transluminal coronary angioplasty (PTCA) or CABG revascularization. Nonetheless, BARI women, despite better left ventricular systolic function, had an excess of periprocedural congestive heart failure or pulmonary edema, with heart failure independently predicting mortality for both women and men. Two issues must be raised: Does the hypertrophied ventricle with diastolic dysfunction tolerate poorly either transient myocardial ischemia or the volume shifts of CABG surgery? Do the extent and pattern of left ventricular hypertrophy differ by sex? Did more optimal myocardial protection in BARI improve the outcome for women undergoing $\mathrm{CABG}$ surgery and what were the characteristics of this protection? Further, since preoperative symptoms of unstable angina and severity of stable angina were greater in women despite less angiographically severe coronary disease, is there a pathophysiologic difference in plaque rupture and/or endothelial dysfunction between women and men-and does this affect perioperative outcomes? In women with fatal coronary thrombosis in another study, plaque characteristics differed between younger and older women. Although, in the overall BARI cohort, CABG offered an advantage compared with PTCA for diabetic patients, the small numbers of women do not allow meaningful comparisons specifically for women. The challenge to the contemporary application of these data involves both the change in percutaneous intervention procedures (including stents and drug-eluting stents) and the change in CABG surgery, with more frequent off-pump procedures, as well as the more aggressive current coronary risk reduction interventions, in part enabled by newer medications.

The Northern New England Cardiovascular Disease Study Group ${ }^{5}$ examined changes in hospital mortality over time for women undergoing CABG surgery, 1987 to 1997. Although in the recent years women were older, had poorer ventricular function, and more often required urgent or emergency CABG surgery, their mortality rates decreased significantly; the absolute magnitude of the change in adjusted mortality rates over time was greater for women than men $(3.1 \%$ vs $1.5 \%)$. Among these almost 30,000 patients, although women represented only $28 \%$ of the study population, the decrease in mortality accounted for $44 \%$ of the total decrease in adjusted mortality during the decade. Why did these older and sicker women do better? Internal thoracic artery grafting increased, but off-pump CABG technique was not used significantly until the end of the study. Did changes in surgical technique improve the approach to smaller coronary vessels in women? Was myocardial protection better? What about approaches to hemodilutional anemia and preinduction high heart rates, which predominated in women? Was there more precise attention to concomitant diabetes, to hypertension? Again, the details of the CABG surgery mortality are not specified. Were deaths operative, perioperative, or late during the hospital stay? What were the specific mechanisms of death?

More women than men presenting for CABG surgery have diabetes. The comparison of diabetic and nondiabetic patients undergoing isolated CABG surgery (1995-1999) showed longer intensive care and hospital stays; greater need for inotropic agents, hemotransfusion, and dialysis; and more frequent renal failure, stroke, mediastinitis, and wound infection. ${ }^{6}$ Patients with insulin-dependent diabetes had even less favorable outcomes. Unfortunately, the report fails to provide sex-specific data. Important questions raised include the role of more intensive insulin treatment and strict blood glucose control.

Information from the National Cardiovascular Network database (1993-1999) was derived from 51,187 patients, $30 \%$ women, who received CABG surgery. Women had higher hospital mortality rates than men, and younger women were at higher risk of hospital death than comparably aged men. ${ }^{7}$ Women younger than age 50 years were 3 times more likely to die than their male counterparts. Women had a greater occurrence of complications, but in particular, renal failure, neurologic complications, and acute myocardial infarction were more marked at younger ages. Again, these younger women not only had more prevalent risk factors but also had more prevalent diabetes. Mechanisms for this excess mortality risk remain obscure, given the less angiographically severe coronary disease and better left ventricular function among the younger women. Again, detailed mortality data require ascertainment.

The Yale-New Haven Hospital database, addressing patients admitted for initial CABG surgery (1999-2001), showed that women had a 6- to 8-week hospital readmission rate of $20.5 \%$ versus $11 \%$ for men. More detailed information might identify the reasons for readmission and suggest interventions likely to curtail these problems. Other recovery comorbid factors for women included an excessive decline in physical functional level and an increase in depressive symptoms. ${ }^{8}$

An abstract report from the Northern New England Cardiovascular Disease Study Group ${ }^{9}$ based on 26,725 consecutive patients undergoing isolated CABG surgery (19932000) showed that greater than $97 \%$ of the excess mortality among women reflected those with diabetes or urgent or emergency presentations. The conclusion was that tight blood glucose control during the perioperative period and excellent medical treatment of acute coronary syndromes, particularly among women, may be important targets for process improvement. Does lesser risk stratification to define and address high risk women during the period of stable angina among women increase the likelihood of urgent or emergency presentation? Women have more severe chest pain and report greater limitation of functional capacity at presentation for CABG surgery. Is the decision for surgery 
inappropriately delayed, initiating a vicious circle? In this cohort, hospital mortality rate was $4.1 \%$ for women versus $2.4 \%$ for men.

\section{What We Must Learn}

Detailed review of available databases should enable delineation of the temporal issues and specific characteristics of the increased perioperative mortality for women. Is this operative mortality, early perioperative mortality, or mortality during the remainder of the hospital stay? Are there sex differences in anesthesia, in pain management? Does preoperative control of coronary risk factors, particularly diabetes, hypertension, and hyperlipidemia, exert an effect? Does mortality differ by age or diabetic status, to name a few factors? An excess of bleeding complications has been described for women with all interventional procedures. Does this play a role in mortality? Is there greater reoperation related to bleeding? What is the role of preoperative anemia?

With hypertrophy of the left ventricle prominent in women, does intraoperative protection against myocardial ischemia play a role? Are the symptoms or other manifestations of perioperative heart failure due to diastolic dysfunction misinterpreted as those owing to ventricular systolic dysfunction and is inappropriate therapy instituted? Is there comparable aggressive pharmacologic risk reduction early in the postoperative phase for women as for men? Are there pathophysiologic differences in coronary plaque and in coronary vasoactivity and/or endothelial function that may contribute to the adverse outcome? How can these be ascertained?

What is the role of precise glucose control, and does it relate to the preoperative as well as the postoperative phase? Does angiotensin-converting enzyme inhibitor therapy, as well as glucose-managing drugs, play a role? Should lipid- lowering drugs be reinstituted earlier? Is this comparable by sex? What is the role of aspirin resumption?

These and multiple other probes of the available data must be designed to ascertain baseline characteristics and to suggest management strategies that may differ by sex. The goal is the elimination or lessening of the sex-based excess morbidity and mortality of CABG surgery, in particular addressing the excess mortality in younger women compared with their male peers.

\section{References}

1. Davis KB, Chaitman B, Ryan T, Bittner V, Kennedy JW. Comparison of 15-year survival for men and women after initial medical or surgical treatment for coronary artery disease: a CASS Registry study. J Am Coll Cardiol. 1995;25:1000-9.

2. Allen JK, Blumenthal RS. Coronary risk factors in women six months after coronary artery bypass grafting. Am J Cardiol. 1995;75:1092-5.

3. Edwards FH, Carey JS, Grover FL, Bero JW, Hartz RS. Impact of gender on coronary bypass operative mortality. Ann Thorac Surg. 1998;66:125-31.

4. Jacobs AK, Kelsey SF, Brooks MM, Faxon DP, Chaitman BR, Bittner $\mathrm{V}$, et al. Better outcome for women compared with men undergoing coronary revascularization. A report from the Bypass Angioplasty Revascularization Investigation (BARI). Circulation. 1998;98:1279-85.

5. O'Rourke DJ, Malenka DJ, Olmstead EM, Quinton HB, Sanders JH Jr, Lahey SJ, et al, for the Northern New England Cardiovascular Disease Study Group. Improved in-hospital mortality in women undergoing coronary artery bypass grafting. Ann Thorac Surg. 2001;71:507-11.

6. Szabó Z, Håkanson E, Svedjeholm R. Early postoperative outcome and medium-term survival in 540 diabetic and 2239 nondiabetic patients undergoing coronary artery bypass grafting. Ann Thorac Surg. 2002; 74:712-9.

7. Vaccarino V, Abramson JL, Veledar E, Weintraub WS. Sex differences in hospital mortality after coronary artery bypass surgery. Evidence for a higher mortality in younger women. Circulation. 2002;105:1176-81.

8. Vaccarino V, Lin ZQ, Kasl SV, Mattera JA, Roumanis SA, Abramson $\mathrm{JL}$, et al. Gender differences in recovery after coronary artery bypass surgery. J Am Coll Cardiol. 2003;41:307-14.

9. O'Connor GT, Morton JR, Olmstead EM, Baribeau YR, Fillinger MP, Cohn WE, et al, for the Northern New England CV Study Group. Excess mortality among women undergoing coronary artery bypass graft surgery [abstract]. Circulation. 2002;106(Suppl):II-552. 